\title{
Hydration requirements in patients receiving highly emetogenic chemotherapy
}

\author{
Randy Erickson*,1, Neil Nebughr ${ }^{1}$, Michael C Mosier ${ }^{2}$ \& William Nibley ${ }^{3}$ \\ ${ }^{1}$ Utah Cancer Specialists, 1121 East 3900 C-230, Salt Lake City, UT 84124, USA \\ ${ }^{2}$ EMB Statistical Solutions, LLC, 55 Corporate Woods, 9300 West 100th Street, Suite 550, Overland Park, KS 66210, USA \\ ${ }^{3}$ Utah Cancer Specialists, 5131 South Cottonwood Street, Suite 200, Murray, UT 84107, USA \\ *Author for correspondence: Tel.: +1 801262 9494; Fax: +1 801267 5623; rerickson@utahcancer.com
}

\begin{abstract}
Aim: Chemotherapy-induced nausea and vomiting diminishes quality of life and increases healthcare resource use. This retrospective medical records analysis evaluated hydration requirements with emetogenic chemotherapy. Patients \& methods: Cancer patients received moderately emetogenic chemotherapy (MEC) or highly emetogenic chemotherapy (HEC), and antiemetics palonosetron or granisetron extendedrelease subcutaneous (GERSC), neurokinin 1 receptor antagonist and dexamethasone. Unscheduled hydration event rates were determined. Results: For 186 patients (92 palonosetron, 94 GERSC) overall, mean hydration rate was significantly higher with palonosetron $(0.6$ vs $0.2 ; p=0.0005)$. Proportion of patients with $\geq 1$ hydration event was significantly higher with palonosetron overall (54 vs $33 \% ; p=0.0033$ ) and in cycles 2-4 and the HEC subgroup. Conclusion: GERSC within a three-drug antiemetic regimen may reduce unscheduled hydration requirements with MEC or HEC.
\end{abstract}

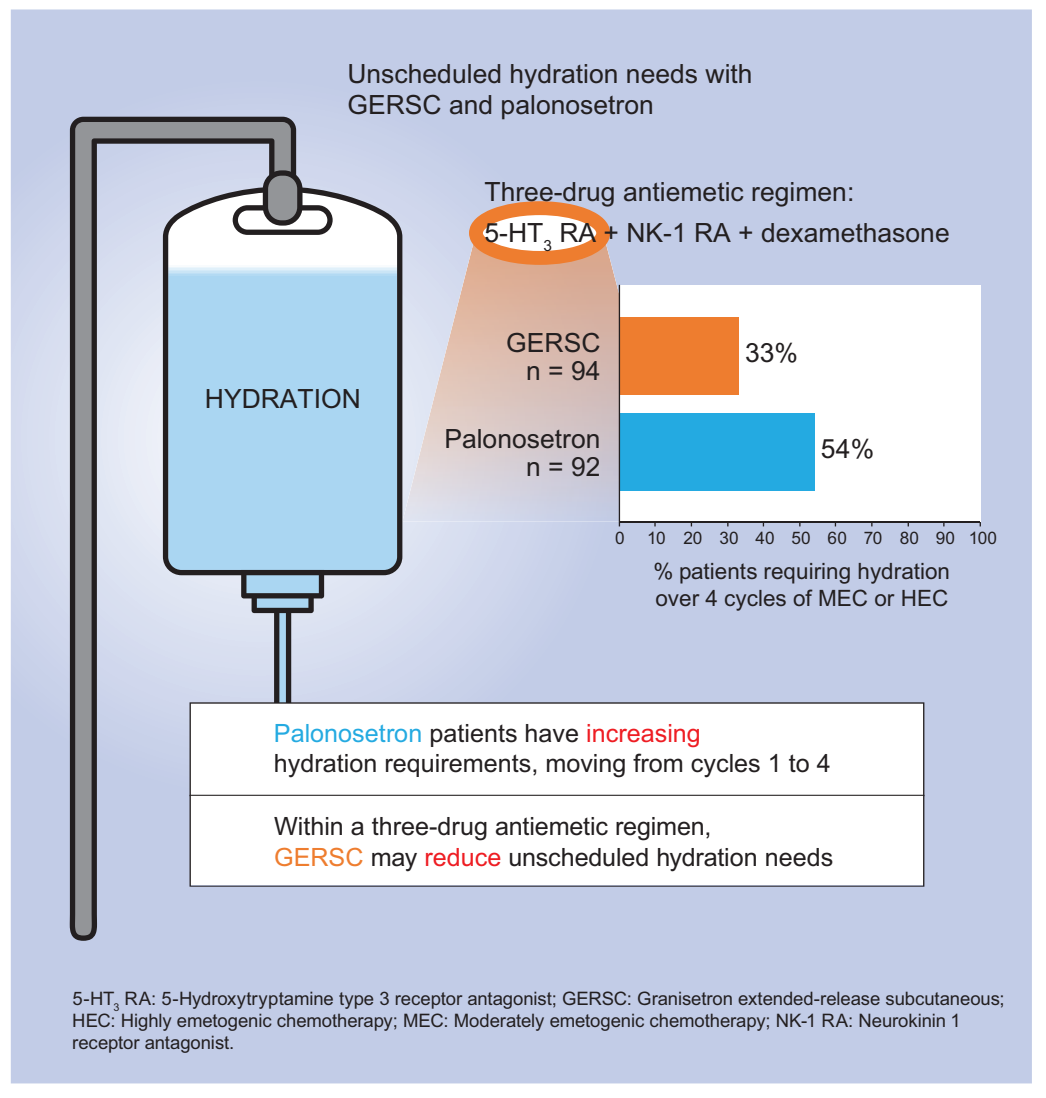

Lay abstract: Chemotherapy-induced nausea and vomiting (CINV) can affect the quality of life and daily functioning of patients with cancer. Additionally, it can lead to increased hospital visits, use of additional 
medications, and needs for hydration with an iv. saline solution. Although guidelines from several organizations recommend combinations of drugs, known as antiemetics, to prevent CINV, there is still a need to improve CINV control. This study evaluated the need for hydration in patients with cancer who were receiving chemotherapy and an antiemetic drug combination including either palonosetron or granisetron extended-release subcutaneous (GERSC). Over half of patients who received palonosetron needed unscheduled hydration, compared with about a third of patients who received GERSC. Also, during each of the four cycles of chemotherapy given, the percentage of patients who needed hydration was higher with palonosetron than with GERSC. These results suggest that GERSC may be preferred over palonosetron in a three-drug antiemetic combination, by reducing the need for unscheduled hydration. This could reduce healthcare costs and improve patients' quality of life.

First draft submitted: 12 October 2018; Accepted for publication: 6 November 2018; Published online: 30 November 2018

Keywords: chemotherapy-induced nausea and vomiting $\bullet$ CINV • extended-release granisetron $\bullet$ highly emetogenic chemotherapy $\bullet$ hydration $\bullet$ moderately emetogenic chemotherapy $\bullet$ palonosetron

Chemotherapy-induced nausea and vomiting (CINV) has significant adverse effects on patients' health, including dehydration, weight loss, metabolic disorders and chemotherapy delay or discontinuation [1], as well as a negative effect on quality of life (QoL) [2,3] and everyday functioning [4]. Additionally, uncontrolled CINV can lead to increased utilization of healthcare resources and incur increased costs [4-7].

Per the National Comprehensive Cancer Network (NCCN) antiemetic guidelines, chemotherapeutic agents are divided into four levels of emetogenic potential (high, moderate, low and minimal) based on the percentage of patients who experience acute emesis when they do not receive antiemetic prophylaxis [1]. For patients receiving moderately emetogenic chemotherapy (MEC) or highly emetogenic chemotherapy (HEC), recommendations from several clinical practice guidelines, such as the NCCN [1], American Society of Clinical Oncology (ASCO) [8], and Multinational Association of Supportive Care in Cancer (MASCC) and European Society of Medical Oncology $[9,10]$, include the use of a three- or four-drug regimen comprising a 5-hydroxytryptamine type $3\left(5-\mathrm{HT}_{3}\right)$ receptor antagonist (RA), a neurokinin 1 (NK-1) RA and dexamethasone $[1,8-10]$, with or without olanzapine (NCCN and ASCO) [1,8]. Per the 2011 guidelines, ASCO reclassified anthracycline (doxorubicin) plus cyclophosphamide (AC) as HEC, previously classified as MEC, given the high emetogenicity of combined agents [11]. The MASCC/European Society of Medical Oncology guidelines provide specific recommendations for CINV prevention in patients with breast cancer who are treated with AC-based chemotherapy [9]. Despite the availability of comprehensive antiemetic guidelines $[1,8-10]$ and advances in antiemetics, there is still a need for more effective CINV prophylaxis [2,3,12,13], and for many patients, CINV prophylaxis remains inadequate [14].

The antiemetic guideline recommendations include the two most commonly used 5- $\mathrm{HT}_{3}$ RAs: palonosetron and granisetron. Palonosetron is a selective $5-\mathrm{HT}_{3} \mathrm{RA}$ approved in the USA in an iv. formulation (Aloxi ${ }^{\circledR}$ ) for CINV prevention in patients receiving MEC or HEC [15]. Granisetron is available in several formulations including transdermal (Sancuso ${ }^{\circledR}$ ) [16] and extended-release subcutaneous (GERSC; Sustol ${ }^{\circledR}$ ) [17]. GERSC was approved in the USA in 2016 for CINV prevention in patients receiving MEC or AC [17]. GERSC is a formulation of $2 \%$ granisetron in a Biochronomer ${ }^{\circledR}$ viscous bioerodible tri(ethylene glycol) poly(orthoester) polymer. A single subcutaneous injection of GERSC provides therapeutic concentrations of granisetron over a 5-day period [18]. In a Phase III noninferiority trial in patients receiving either MEC or HEC, GERSC $500 \mathrm{mg}$ was noninferior to palonosetron $0.25 \mathrm{mg}$, both in combination with dexamethasone, for the prevention of acute and delayed CINV in patients with cancer receiving MEC and noninferior to palonosetron in the acute phase in patients receiving HEC $[19,20]$. Adverse events reported in both treatment groups were mild, and most were considered unrelated to treatment. The most common treatment-related injection-site reaction was bruising, and although treatmentrelated injection-site reactions were more frequent in the GERSC group than in the palonosetron group, they were mild and resolved overtime [19,20]. The registrational Phase III MAGIC trial evaluated the antiemetic efficacy and safety of GERSC compared with the 5- $\mathrm{HT}_{3} \mathrm{RA}$ ondansetron as part of a three-drug regimen including the NK-1 RA fosaprepitant and dexamethasone in patients with cancer receiving HEC [21]. GERSC was significantly superior to ondansetron in eliciting a complete response ( 65 vs $57 \% ; \mathrm{p}=0.014$ ), defined as no emesis or use of rescue medication, in the delayed phase of CINV. Additionally, GERSC, compared with ondansetron, significantly 
reduced the use of rescue medication in delayed (71 vs 63\%; $\mathrm{p}=0.013)$ and overall phases (66 vs 60\%; $\mathrm{p}=0.038$ ), with fewer patients experiencing nausea across the study period [21].

The management of uncontrolled or poorly controlled CINV imposes an economic burden with respect to increases in hospitalization, use of rescue medications and use of hydration therapy, as well as affecting patients' productivity and potential for missed days at work [7,22]. This retrospective study was conducted to evaluate unscheduled hydration requirements in patients with cancer receiving MEC or HEC and a prophylactic antiemetic regimen that included either palonosetron or GERSC as the $5-\mathrm{HT}_{3} \mathrm{RA}$.

\section{Patients \& methods}

\section{Patients \& treatments}

Medical records were assessed for patients with cancer who received MEC or HEC and a recommended three-drug antiemetic regimen that included either palonosetron or GERSC as the $5-\mathrm{HT}_{3} \mathrm{RA}$ in combination with an $\mathrm{NK}-1$ RA (fosaprepitant iv.) and dexamethasone at a single community-based practice with nine locations. Demographic data were analyzed, including patient age, sex, cancer diagnosis, emetogenic potential of the chemotherapy regimen (MEC or HEC) administered as defined by NCCN guidelines [1], antiemetic regimen and number of required unscheduled hydration events during chemotherapy cycles.

\section{Study objective}

This retrospective analysis was performed to determine the proportion of patients who required unscheduled hydration related to CINV during chemotherapy in the two antiemetic regimen groups (palonosetron or GERSC) overall, by cycle, and by emetogenicity of their chemotherapy regimens. Comparisons were made between patients who received palonosetron and patients who received GERSC, each as part of a three-drug regimen, which included fosaprepitant iv. and dexamethasone.

\section{Statistical analyses}

Patients treated with palonosetron received no more than four chemotherapy cycles, while 26 patients treated with GERSC received more than four chemotherapy cycles. Since the risk of hydration requirements increases as the number of cycles increases, only data from cycles 1-4 were used in the analyses to make the treatment groups comparable. The use of unscheduled hydration was computed as the hydration rate, or number of unscheduled hydration events, divided by the total number of chemotherapy cycles. A nonparametric two-sided Wilcoxon rank sum test with $\alpha=0.05$ was used to test the null hypothesis that there was no difference between groups in the overall hydration rate, since the distribution of the rate variable is extremely right skewed. Also, for analyses conducted separately for each cycle, the number of hydration events in that cycle was the dependent variable, and comparisons were made using the same nonparametric two-sided Wilcoxon rank sum test. Additionally, the $\chi^{2}$ test was used to compare the percentage of patients who required any hydration, overall and separately by chemotherapy cycle.

\section{Results}

\section{Patient disposition \& characteristics}

The patient population included 92 patients who were treated with palonosetron and 94 patients, who were treated with GERSC from January 2016 until December 2017. Of the total 186 patients who received MEC or HEC, only 17 patients received MEC (one patient received palonosetron, 16 patients received GERSC) (Table 1). Therefore, presented here are overall analyses, from cycles 1-4 of chemotherapy, for all patients (MEC and HEC) and subgroup analyses only for patients who received HEC.

Patient demographics and clinical characteristics are shown in Table 1. Most patients in each antiemetic treatment group were women (palonosetron 99\%, GERSC 74\%; p < 0.001), and the most common cancer diagnosis was breast cancer (palonosetron 82\%, GERSC 40\%; p < 0.001). All patients received fosaprepitant $150 \mathrm{mg}$ iv. and dexamethasone $10 \mathrm{mg}$ in addition to either palonosetron $0.25 \mathrm{mg}$ iv. or GERSC $10 \mathrm{mg}$ SC on day 1 (Table 1). Patients were also prescribed oral dexamethasone three-times daily.

The most common chemotherapy regimens were a dose-dense anthracycline (doxorubicin) and cyclophosphamide (DDAC)-based regimen in the patients receiving palonosetron $(\mathrm{n}=46,50 \%)$ and a DDAC regimen in the patients receiving GERSC $(n=25,27 \%)$ (Table 2). The AC subgroup of patients comprised 73 patients treated with palonosetron and 25 with GERSC who received DDAC-based chemotherapy (Table 2). 


\begin{tabular}{|c|c|c|c|}
\hline Demographic or characteristic & Palonosetron $\mathrm{n}=92$ & GERSC $n=94$ & p-value \\
\hline Age, mean (min, max), y & $55(28,79)$ & $64(21,87)$ & $<0.001$ \\
\hline Sex, n (\%) & & & $<0.001$ \\
\hline- Men & $1(1)$ & $24(26)$ & \\
\hline - Women & $91(99)$ & $70(74)$ & \\
\hline Cancer type, n (\%) & & & $<0.001$ \\
\hline - Breast & $75(82)$ & $38(40)$ & \\
\hline - Ovarian & $7(8)$ & $15(16)$ & \\
\hline - Others & $10(11)$ & $41(44)$ & \\
\hline \multicolumn{4}{|c|}{ NCCN emetogenicity classification, $n(\%)$} \\
\hline$-\mathrm{HEC}, \mathrm{n}(\%)$ & $91(99)$ & $78(83)$ & $<0.001$ \\
\hline$-\mathrm{MEC}, \mathrm{n}(\%)$ & $1(1)$ & $16(17)$ & \\
\hline Antiemetic regimen & $\begin{array}{l}\text { Palonosetron } 0.25 \mathrm{mg} \text { iv. + fosaprepitant } \\
150 \mathrm{mg} \text { iv. + dexamethasone } 10 \mathrm{mg}^{\S}\end{array}$ & $\begin{array}{l}\text { GERSC } 10 \mathrm{mg} \\
+ \text { dexamethas }\end{array}$ & \\
\hline \multicolumn{4}{|c|}{ 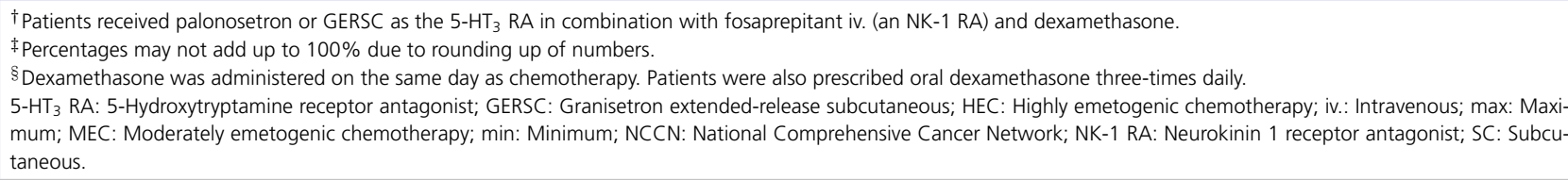 } \\
\hline
\end{tabular}

\section{Table 2. Distribution of chemotherapy regimens administered.}

\begin{tabular}{|c|c|c|}
\hline Regimen & Palonosetron $(n=92)$ & GERSC $(n=94)$ \\
\hline MEC regimens, $\mathrm{n}(\%)$ : & $1(1)$ & $16(17)$ \\
\hline - Bendamustine + rituximab & - & $2(2)$ \\
\hline - Cisplatin & $1(1)$ & - \\
\hline - FOLFOX & - & $10(11)$ \\
\hline - Docetaxel + cyclophosphamide & - & $3(3)$ \\
\hline HEC regimens, $n(\%)$ : & 91 (99) & $78(83)$ \\
\hline - Doxorubicin + olaratumab & - & $1(1)$ \\
\hline - Bendamustine + rituximab & - & $4(4)$ \\
\hline - Carboplatin AUC $5+$ cetuximab & $1(1)$ & - \\
\hline - Carboplatin + paclitaxel & $15(16)$ & $1(1)$ \\
\hline - Carboplatin + docetaxel & - & $2(2)$ \\
\hline - Carboplatin AUC 5 + gemcitabine & - & $1(1)$ \\
\hline - Carboplatin AUC 6 + paclitaxel & - & $17(18)$ \\
\hline - Carboplatin AUC 6 + etoposide & - & $2(2)$ \\
\hline - Cisplatin + etoposide & - & $1(1)$ \\
\hline - Cisplatin & $1(1)$ & $8(9)$ \\
\hline- DDAC & $21(23)$ & $25(27)$ \\
\hline - DDAC + weekly paclitaxel & $46(50)$ & - \\
\hline - DDAC + paclitaxel & $6(7)$ & - \\
\hline - Docetaxel & $1(1)$ & - \\
\hline - Docetaxel + cyclophosphamide & - & $6(6)$ \\
\hline - Docetaxel + cyclophosphamide + trastuzumab & - & $3(3)$ \\
\hline$-\mathrm{R}-\mathrm{CHOP}$ & - & $2(2)$ \\
\hline
\end{tabular}




\begin{tabular}{|c|c|c|c|c|c|}
\hline \multirow[t]{2}{*}{ Treatment } & \multicolumn{2}{|c|}{ Number of chemotherapy cycles } & \multicolumn{2}{|c|}{ Hydration rate } & \multirow{2}{*}{$\begin{array}{l}\text { p-value (Wilcoxon rank } \\
\text { sum) }\end{array}$} \\
\hline & Mean (SD) & Median (min, max) & Mean (SD) & Median (min, max) & \\
\hline \multicolumn{6}{|l|}{ Total } \\
\hline Palonosetron $(n=92)$ & $3.9(0.5)$ & $4.0(1,4)$ & $0.6(1.1)$ & $0.3(0,5.8)$ & 0.0005 \\
\hline GERSC $(n=94)$ & $3.7(0.5)$ & $4.0(2,4)$ & $0.2(0.4)$ & $0(0,2.3)$ & \\
\hline \multicolumn{6}{|l|}{ HEC } \\
\hline Palonosetron $(n=91)$ & $3.9(0.5)$ & $4.0(1,4)$ & $0.6(1.1)$ & $0.3(0,5.8)$ & 0.0007 \\
\hline GERSC $(n=78)$ & $3.7(0.5)$ & $4.0(2,4)$ & $0.2(0.4)$ & $0(0,2.3)$ & \\
\hline \multicolumn{6}{|l|}{ AC } \\
\hline Palonosetron $(n=73)$ & $3.9(0.6)$ & $4.0(1,4)$ & $0.6(0.9)$ & $0.3(0,5.0)$ & 0.0338 \\
\hline GERSC $(n=25)$ & $3.6(0.7)$ & $4.0(2,4)$ & $0.2(0.4)$ & $0(0,1.8)$ & \\
\hline
\end{tabular}

$\dagger$ Cycles 1-4 only. Patients treated with palonosetron received no more than four chemotherapy cycles, while 26 patients treated with GERSC received more than four chemotherapy cycles. Only data from the first four cycles were used in the analyses to make the treatments comparable.

AC: Anthracycline (doxorubicin) plus cyclophosphamide; GERSC: Granisetron extended-release subcutaneous; HEC: Highly emetogenic chemotherapy; max: Maximum; min: Minimum.

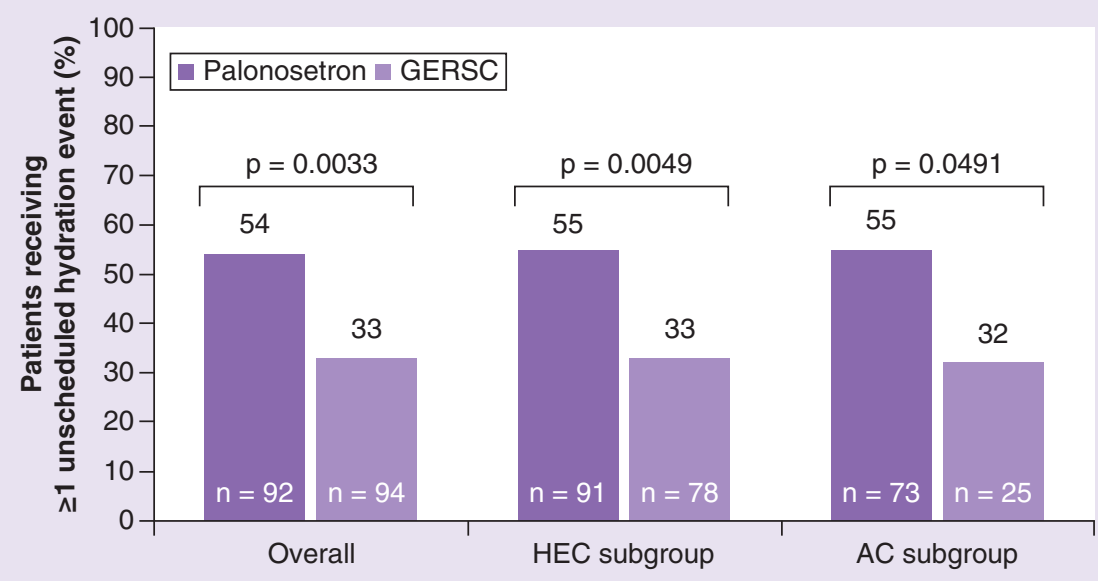

Figure 1. Proportion of overall population, highly emetogenic chemotherapy subgroup and anthracycline (doxorubicin) plus cyclophosphamide subgroup with at least one unscheduled hydration event over chemotherapy cycles 1-4.

AC: Anthracycline (doxorubicin) plus cyclophosphamide; GERSC: Granisetron extended-release subcutaneous; HEC: Highly emetogenic chemotherapy.

For all patients receiving palonosetron, the mean (SD) number of chemotherapy cycles was 3.9 (0.5), and for patients receiving GERSC, it was 3.7 (0.5) (Table 3). The numbers remained the same for the HEC subgroup (Table 3). In the AC subgroup, the mean (SD) number of chemotherapy cycles was 3.9 (0.6) with palonosetron and 3.6 (0.7) with GERSC (Table 3).

\section{Unscheduled hydration analyses}

Each hydration event was typically $1000 \mathrm{ml}$ of normal saline administered iv. over $60 \mathrm{~min}$, with or without additional antiemetics such as ondansetron or metoclopramide plus diphenhydramine. The mean (SD) unscheduled hydration rate was significantly higher for all patients receiving palonosetron versus all patients receiving GERSC (0.6 [1.1] vs 0.2 [0.4], respectively; $\mathrm{p}=0.0005$ ) (Table 3 ). Findings were similar for the HEC subgroup, where the hydration rate was significantly higher with palonosetron than with GERSC (0.6 [1.1] vs 0.2 [0.4], respectively; $\mathrm{p}=0.0007)$ (Table 3), and for the AC subgroup, where the hydration rate was significantly higher with palonosetron than with GERSC (0.6 [0.9] vs 0.2 [0.4], respectively; $\mathrm{p}=0.0338$ ) (Table 3).

The proportion of patients who had at least one hydration event was significantly higher with palonosetron than with GERSC (54 vs 33\%, respectively; $\mathrm{p}=0.0033$ ) (Figure 1). Similar significant results were found for the HEC 


\begin{tabular}{|c|c|c|c|c|c|c|}
\hline \multirow[t]{2}{*}{ Cycle } & \multicolumn{2}{|c|}{ Patients receiving at least one hydration } & \multirow[t]{2}{*}{ p-value $\left(\chi^{2}\right)$} & \multicolumn{2}{|c|}{ Rate of unscheduled hydration } & \multirow{2}{*}{$\begin{array}{l}\text { p-value (Wilcoxon rank } \\
\text { sum) }\end{array}$} \\
\hline & $\begin{array}{l}\text { Palonosetron } \\
(n=91)^{\dagger}\end{array}$ & GERSC $(n=94)$ & & $\begin{array}{l}\text { Palonosetron } \\
(n=91)^{\dagger}\end{array}$ & GERSC $(n=94)$ & \\
\hline 1 & $21(23)$ & $14(15)$ & 0.1554 & 0.4 & 0.2 & 0.1315 \\
\hline 2 & $24(26)$ & 11 (12) & 0.0109 & 0.5 & 0.1 & 0.0079 \\
\hline 3 & $25(28)$ & $14(15)$ & 0.0360 & 0.6 & 0.2 & 0.0252 \\
\hline 4 & $36(40)$ & $15(16)$ & 0.0003 & 0.8 & 0.2 & 0.0002 \\
\hline
\end{tabular}

subgroup and the AC subgroup (Figure 1). In each of cycles 1-4, the proportion of overall patients who required at least one hydration event was greater with palonosetron than with GERSC (Table 4). Although this difference was not significant in cycle 1 , it was significant in cycles $2(p=0.0109), 3(p=0.0360)$ and $4(p=0.0003)$. The greatest difference occurred in cycle 4: $40 \%$ of patients receiving palonosetron required at least one hydration event, versus $16 \%$ receiving GERSC (Table 4). Similar results were observed with the HEC subgroup analysis of hydration with palonosetron versus GERSC (Supplementary Table 1). For the AC subgroup, in each of cycles 1-4, the percentage of patients who required at least one hydration event was greater with palonosetron than with GERSC. However, this difference was significant only in cycle $4(\mathrm{p}=0.0205)$ (Supplementary Table 2).

\section{Discussion}

This retrospective analysis evaluated the medical records of patients who received either HEC or MEC and a recommended three-drug antiemetic regimen that included a 5-HT 3 RA, the NK-1 RA, fosaprepitant and dexamethasone. Patients were treated with either palonosetron or GERSC as the 5- $\mathrm{HT}_{3} \mathrm{RA}$. The primary objective of this study was to evaluate the CINV-related hydration requirements of these patients during each cycle of chemotherapy.

In the overall population who received HEC or MEC, the most common diagnosis was breast cancer. More than $50 \%$ of patients (HEC or MEC) who received palonosetron required at least one unscheduled hydration event over cycles 1-4 of chemotherapy, compared with approximately a third of patients who received GERSC, a significant difference in favor of GERSC. During each of cycles 1-4, the percentage of patients who required at least one hydration event was higher with palonosetron than with GERSC, a difference that was significant in cycles 2-4. The percentage of patients requiring hydration was highest during cycle 4, in those receiving palonosetron. Findings were similar in the HEC subgroup. Hydration requirements showed similar trends in the AC subgroup during cycles $1-3$, although in cycle 4 a significantly higher percentage of patients receiving palonosetron required at least one hydration event.

Limitations of the current study include that it was retrospective and not designed for equal inclusion of demographic data; therefore, there is an imbalance between the groups of patients receiving palonosetron and those receiving GERSC. This imbalance was also partly due to the payers indicating the drug to be used. Also, since only 17 patients received MEC, only one of whom received palonosetron, no analyses were done for the MEC subgroup. However, in a prospective observational study evaluating the impact of guideline-consistent three-drug antiemetic prophylaxis, responses (no emesis, no clinically significant nausea) were comparable for patients receiving HEC or MEC [13].

Considerable advances have been made in the availability of antiemetics, and guideline recommendations now include several options for effective antiemetic regimens. However, effective CINV prevention remains a challenge. Not only does poorly managed CINV reduce patients' QoL, it can also lead to greater utilization of healthcare resources and increase the cost of care [7]. Several studies have shown the economic burden of poorly controlled CINV [5,7,23,24]. The increased costs can be attributed to increased inpatient and outpatient visits, increased emergency room visits and the use of rescue medications [5,7,24]. These findings were confirmed by a recent report from the US Agency for Healthcare Research and Quality's Healthcare Cost and Utilization Project, which reported 37,730 hospital discharges associated with nausea and vomiting, with each event estimated to cost US $\$ 11,232$ and the total hospitalization cost estimated to be US $\$ 15,120$ per discharge [22]. Consequently, in the current retrospective study, the increase in unscheduled hydration requirements in patients receiving palonosetron 
compared with GERSC (each as part of a three-drug antiemetic regimen) would likely increase healthcare costs for patients who receive palonosetron as the $5-\mathrm{HT}_{3} \mathrm{RA}$ in a three- or four-drug antiemetic regimen. Although this was a retrospective review of medical records at a single practice with multiple sites, similar results were reported in another retrospective study, in which 1.5 to two-times more patients required hydration events when receiving palonosetron than with GERSC (each as part of a three-drug antiemetic regimen) [25]. Further, in this analysis, for the HEC subgroup, approximately three-times more hydration events were required in patients treated with palonosetron than with GERSC. Together, these findings suggest that GERSC may be preferred over palonosetron as the $5-\mathrm{HT}_{3}$ $\mathrm{RA}$ of choice in a three-drug antiemetic regimen, by reducing unscheduled hydration requirements [25]. A reduction in the number of hydration events required would be expected to decrease healthcare costs and improve patients' daily functioning and QoL.

\section{Conclusion}

A three-drug antiemetic regimen including GERSC, rather than palonosetron, may lead to a reduction in the number hydration events required by patients receiving MEC or HEC.

\section{Summary points}

- Chemotherapy-induced nausea and vomiting (CINV) adversely affects patients' health, quality of life and daily functioning.

- Poorly controlled CINV may lead to an economic burden on patients as well as increased utilization of healthcare resources.

- Despite recommendations from several comprehensive antiemetic guidelines, there remains a need for more effective CINV prophylaxis.

- This current, retrospective study evaluated the CINV-related hydration requirements in patients with cancer receiving moderately emetogenic chemotherapy (MEC) or highly emetogenic chemotherapy (HEC) and a guideline-recommended antiemetic regimen that includes the 5-hydroxytrypamine $\left(5-\mathrm{HT}_{3}\right)$ receptor antagonist (RA) palonosetron or granisetron extended-release subcutaneous (GERSC), in combination with a neurokinin 1 RA (fosaprepitant iv.) and dexamethasone.

- Greater than $50 \%$ of patients (MEC or HEC) receiving palonosetron required at least one unscheduled hydration event compared with approximately a third of patients receiving GERSC, over cycles 1-4 of chemotherapy.

- A significantly greater percentage of patients receiving palonosetron required unscheduled hydration in cycles 2-4 compared with patients receiving GERSC.

- Results were similar in the HEC subgroup of patients, with a similar trend observed in patients in the anthracycline plus cyclophosphamide subgroup.

- GERSC may be preferred over palonosetron as the 5- $\mathrm{HT}_{3} \mathrm{RA}$ of choice within a three-drug antiemetic regimen; GERSC has lower unscheduled hydration requirements compared with palonosetron, potentially decreasing healthcare costs and improving patients' quality of life and functioning.

Supplementary data

To view the supplementary data that accompany this paper please visit the journal website at: www.futuremedicine.com/doi/suppl/10.2217/fon-2018-0787

Financial \& competing interests disclosure

R Erickson reports employment by and leadership role at Utah Cancer Specialists; has served as a consultant/advisor for, been part of a speakers bureau, received honoraria from, and compensation for travel and accommodations from Heron. N Nebughr has served as a consultant/advisor for, been part of a speakers bureau for, and received honoraria from Heron. MC Mosier has served as a consultant/advisor for Heron; W Nibley reports a leadership role at Utah Cancer Specialists and has received honoraria from AstraZeneca, Heron, and Tesaro. Statistical analysis for this study was funded by Heron Therapeutics, Inc., CA, USA. The authors have no other relevant affiliations or financial involvement with any organization or entity with a financial interest in or financial conflict with the subject matter or materials discussed in the manuscript apart from those disclosed.

Medical writing support was provided by N Parikh, SciStrategy Communications, and funded by Heron Therapeutics, Inc. 
Open access

This work is licensed under the Attribution-NonCommercial-NoDerivatives 4.0 Unported License. To view a copy of this license, visit http://creativecommons.org/licenses/by-nc-nd/4.0/

\section{References}

Papers of special note have been highlighted as: $\bullet$ of interest; $\bullet \bullet$ of considerable interest

1. National Comprehensive Cancer Network. NCCN Clinical Practice Guidelines in Oncology: Antiemesis v3.2018. www.nccn.org/professionals/physician_gls/default.aspx

- US clinical practice guidelines for the prevention and treatment of chemotherapy-induced nausea and vomiting (CINV).

2. Hilarius DL, Kloeg PH, Van Der Wall E, Van Den Heuvel JJ, Gundy CM, Aaronson NK. Chemotherapy-induced nausea and vomiting in daily clinical practice: a community hospital-based study. Support. Care Cancer 20(1), 107-117 (2012).

3. Bloechl-Daum B, Deuson RR, Mavros P, Hansen M, Herrstedt J. Delayed nausea and vomiting continue to reduce patients' quality of life after highly and moderately emetogenic chemotherapy despite antiemetic treatment. J. Clin. Oncol. 24(27), 4472-4478 (2006).

4. Haiderali A, Menditto L, Good M, Teitelbaum A, Wegner J. Impact on daily functioning and indirect/direct costs associated with chemotherapy-induced nausea and vomiting (CINV) in a U.S. population. Support. Care Cancer 19(6), 843-851 (2011).

5. Burke TA, Wisniewski T, Ernst FR. Resource utilization and costs associated with chemotherapy-induced nausea and vomiting (CINV) following highly or moderately emetogenic chemotherapy administered in the US outpatient hospital setting. Support. Care Cancer 19(1), 131-140 (2011).

6. Shih TYC, Xu Y, Elting LS. Costs of uncontrolled chemotherapy-induced nausea and vomiting among working-age cancer patients receiving highly or moderately emetogenic chemotherapy. Cancer 110(3), 678-685 (2007).

7. Sommariva S, Pongiglione B, Tarricone R. Impact of chemotherapy-induced nausea and vomiting on health-related quality of life and resource utilization: a systematic review. Crit. Rev. Oncol. Hematol. 99, 13-36 (2016).

- Comprehensive review outlining evidence of the effect of CINV on patients' quality of life and its healthcare cost burden.

8. Hesketh PJ, Kris MG, Basch E et al. Antiemetics: American Society of Clinical Oncology clinical practice guideline update. J. Clin. Oncol. 35(28), 3240-3261 (2017).

- US clinical practice guidelines for the prevention and treatment of CINV.

9. Herrstedt J, Roila F, Warr D et al. 2016 Updated MASCC/ESMO consensus recommendations: prevention of nausea and vomiting following high emetic risk chemotherapy. Support. Care Cancer 25(1), 277-288 (2017).

10. Roila F, Warr D, Hesketh PJ et al. 2016 updated MASCC/ESMO consensus recommendations: prevention of nausea and vomiting following moderately emetogenic chemotherapy. Support. Care Cancer 25(1), 289-294 (2017).

- European clinical practice guidelines for the prevention and treatment of nausea and vomiting induced by moderately emetogenic chemotherapy.

11. Basch E, Prestrud AA, Hesketh PJ et al. Antiemetics: American Society of Clinical Oncology clinical practice guideline update. J. Clin. Oncol. 29(31), 4189-4198 (2011).

12. Clark-Snow R, Affronti ML, Rittenberg CN. Chemotherapy-induced nausea and vomiting (CINV) and adherence to antiemetic guidelines: results of a survey of oncology nurses. Support. Care Cancer 26(2), 557-564 (2018).

13. Gilmore JW, Peacock NW, Gu A et al. Antiemetic guideline consistency and incidence of chemotherapy-induced nausea and vomiting in US community oncology practice: INSPIRE Study. J. Oncol. Pract. 10(1), 68-74 (2014).

14. De Laurentiis $\mathrm{M}$, Bonfadini $\mathrm{C}$, Lorusso $\mathrm{V}$ et al. Incidence of nausea and vomiting in breast cancer patients treated with anthracycline plus cyclophosphamide-based chemotherapy regimens in Italy: NAVY observational study. Support. Care Cancer 26, 4021-4029 (2018).

15. Aloxi (palonosetron $\mathrm{HCl}$ ) injection, for intravenous use [prescribing Information]. Heron Therapeutics, Redwood City, CA, USA (2015).

16. Sancuso (granisetron transdermal system) [prescribing Information]. Kyowa Kirin, Bedminster, NJ, USA (2017).

17. Sustol (granisetron) extended-release injection, for subcutaneous use [prescribing Information]. Heron Therapeutics, Redwood City, CA, USA (2017).

18. Ottoboni T, Gelder MS, O'Boyle E. Biochronomer technology and the development of APF530, a sustained release formulation of granisetron. J. Exp. Pharmacol. 6, 15-21 (2014).

19. Raftopoulos H, Boccia R, Cooper W, O’Boyle E, Gralla RJ. Slow-release granisetron (APF530) versus palonosetron for chemotherapy-induced nausea/vomiting: analysis by American Society of Clinical Oncology emetogenicity criteria. Future Oncol. 11(18), 1-11 (2015).

20. Raftopoulos H, Cooper W, O’Boyle E, Gabrail N, Boccia R, Gralla RJ. Comparison of an extended-release formulation of granisetron (APF530) versus palonosetron for the prevention of chemotherapy-induced nausea and vomiting associated with moderately or highly emetogenic chemotherapy: results of a prospective, randomized, double-blind, noninferiority Phase 3 trial. Support. Care Cancer 23(3), 723-732 (2015). 
21. Schnadig ID, Agajanian R, Dakhil SR et al. APF530 (granisetron inj extended-release) in a three-drug regimen for delayed CINV in highly emetogenic chemotherapy. Future Oncol. 12(12), 1469-1481 (2016).

-. Phase III trial of granisetron extended-release subcutaneous versus ondansetron, each in a three-drug regimen, for the prevention of delayed CINV in patients receiving highly emetogenic chemotherapy.

22. Roeland E, Ma J, Binder G et al. Hospitalization costs for nausea and vomiting: a savings opportunity. J. Clin. Oncol. 35(31 Suppl.), 155-155 (2017).

23. Ballatori E, Roila F, Ruggeri B et al. The cost of chemotherapy-induced nausea and vomiting in Italy. Support. Care Cancer 15(1), 31-38 (2007).

24. Craver C, Gayle J, Balu S, Buchner D. Clinical and economic burden of chemotherapy-induced nausea and vomiting among patients with cancer in a hospital outpatient setting in the United States. J. Med. Econ. 14(1), 87-98 (2011).

25. Vacirca J, Caruana D, Calcanes G, Mosier M, Boccia R, McBride A. Hydration requirements with emetogenic chemotherapy: granisetron extended-release subcutaneous versus palonosetron. Future Oncol. 14(14), 1387-1396 (2018). 
